\title{
Predictors of abnormalities on magnetic resonance cholangiopancreatography: is there a role when the biliary tree is normal on previous imaging?
}

\author{
Bhamini Vadhwana a , John Grabya , Martin Lewis ${ }^{a}$, Anouchka Goldmana , Antoni Sergot ${ }^{\mathrm{b}}$, \\ Catherine Ramsey ${ }^{b}$, Kevin J. Monahana
}

West Middlesex University Hospital, Isleworth, UK

Abstract

\begin{abstract}
Background There is limited evidence supporting the use of magnetic resonance cholangiopancreatography (MRCP) if the biliary tree is within normal limits on ultrasound scan (US) or computed tomography (CT). The aim of this study was to assess the role of MRCP in the absence of a dilated biliary system on index imaging.
\end{abstract}

Methods A retrospective observational study of consecutive MRCP investigations ( $n=427)$ was performed between October 2010 and June 2013 at a single district general hospital. Data collected included patient demographics, clinical presentation, liver function tests (LFTs) and radiological presence of stones. Binary logistic regression and chi-square test were performed using SPSS v23.

Results We included 358 cases, $65 \%$ female $(n=231)$ and $35 \%$ male $(n=127)$, with a mean age of 60 years. Of these, $63 \%$ presented with abdominal pain $(n=225)$, with $20 \%$ having concurrent deranged LFTs $(n=44)$ and $8 \%$ jaundice $(n=18)$. Index imaging demonstrated a dilated biliary system $>6 \mathrm{~mm}$ in $68 \%(\mathrm{n}=245)$. Alkaline phosphatase (ALP) elevation was an independent positive predictor for an abnormal MRCP $(\mathrm{P}=0.003)$. Abnormal index imaging, ALP and clinical jaundice were all significantly associated with a positive $\mathrm{MRCP}(\mathrm{P}<0.001, \mathrm{P}=0.028, \mathrm{P}=0.018)$.

Conclusions It is efficacious to proceed to MRCP with abnormal findings on index imaging, clinical jaundice or elevated ALP. An MRCP scan should be strongly considered in the context of elevated ALP and normal US/CT biliary system.

Keywords Magnetic resonance cholangiopancreatography, abnormal biliary tree, cholelithiasis, alkaline phosphatase, bilirubin

Ann Gastroenterol 2019; 32 (2): 1-6

\section{Introduction}

Cholelithiasis, or gallstone disease, is an increasingly common gastrointestinal disease in the adult population, with a UK prevalence of $15 \%$ [1]. Known predictive risk factors

Department of ${ }^{\mathrm{a}}$ Gastroenterology (Bhamini Vadhwana, John Graby, Martin Lewis, Anouchka Goldman, Kevin J. Monahan); ${ }^{\text {RRadiology }}$ (Antoni Sergot, Catherine Ramsey), West Middlesex University Hospital, Twickenham Road, Isleworth TW7 6AF, UK

\section{Conflict of Interest: None}

Correspondence to: Bhamini Vadhwana, Department of Gastroenterology, West Middlesex University Hospital, Twickenham Road, Isleworth TW7 6AF, UK, e-mail: bhaminivadhwana@doctors.org.uk

Received 19 August 2018; accepted 17 December 2018; published online 15 January 2019

DOI: https://doi.org/10.20524/aog.2019.0352 include female sex, positive family history, a calorific diet high in unsaturated fats, pregnancy and associated conditions such as hypercholesterolemia, diabetes mellitus and obesity [2]. With an aging population and an increasing average body mass index, the morbidity and mortality associated with gallstone disease is a growing problem.

For the majority of individuals, gallstone disease is an incidental finding after imaging of the abdomen for unrelated reasons or investigation for abnormal liver function tests (LFTs). Although they remain asymptomatic, $15-26 \%$ are expected to become symptomatic within 10 years, with $10-20 \%$ developing choledocholithiasis, otherwise commonly referred to as ductal stones $[1,3]$. A watch-and-wait approach is adopted for incidental asymptomatic stones within the gallbladder; however, it is important to be aware of the onset of biliary colic and the risk of complications such as acute cholecystitis and those associated with choledocholithiasis [4]. This includes obstructive jaundice, cholangitis, acute pancreatitis, and chronic disease such as biliary cirrhosis or portal hypertension. 
Transabdominal ultrasound scanning (US) is recognized as the gold standard first-line investigation $[1,5]$. All reports should comment on dilatation of the biliary system, even if it is negative. If there is further suspicion of ductal stones in cases of abnormal LFTs or an US-confirmed dilated common bile duct (CBD), according to UK guidelines magnetic resonance cholangiopancreatography (MRCP) should be performed [1]. Computed tomography (CT) is not commonly the investigation of choice, with a sensitivity of $50-88 \%$ and specificity of $84-98 \%$, but may be used in the acute scenario [6].

The National Institute for Clinical Excellence (NICE) guidelines recommend the use of MRCP in cases of abnormal LFTs or a dilated CBD, however, its role is unclear in the absence of a dilated biliary tree. Clinically, MRCP may be requested in cases of persistent pain or abnormal LFTs after cholecystectomy [5,7]. Given the increasing strain on the National Health Service (NHS) from recurrent hospitalizations and provision of resources, the objective of our study was to investigate the role and determine the diagnostic yield of MRCP in the absence of a dilated biliary system.

\section{Patients and methods}

We carried out a retrospective observational study of consecutive MRCP investigations $(n=427)$ performed between October 2010 and June 2013 at a district general hospital. Data collection was performed independently by three researchers and included patient demographics (age and sex); clinical presentation, primarily pain and jaundice; and serum LFTs including bilirubin (1-20 $\mu \mathrm{mol} / \mathrm{L})$, alkaline phosphatase (ALP; 25-110 IU/L), alanine transaminase (ALT; 5-45 IU/L), aspartate aminotransferase (AST; 5-45 IU/L) and $\gamma$-glutamyltransferase (GGT; $<65 \mathrm{IU} / \mathrm{L})$. Index scan findings (US or CT) were evaluated for the presence or absence of stones, CBD dilatation and intrahepatic duct dilatation. Initial US and CT scan findings were correlated with subsequent MRCP findings. The MRCP images were obtained using a Phillips Achieva 1.5T (2010). The patient was fasted for $4 \mathrm{~h}$ and advised to take sips of water prior to the investigation. The MRCP protocol used at our institution consists of a T2 HASTE (long TE) axial, T2 TSE Axial, T1 TFE Axial, T1 TFE coronal, SSh_MRCP rad radial slab MRCP and SMRCP_3D_HR thin segment MRCP. All MRCPs were reported by a consultant radiologist with a specialist interest in hepatobiliary imaging. The images were reported off an Agfa PACS workstation.

The definition of a "dilated" CBD was taken from the accepted radiology rule, as stated by Laing, to "consider as normal a $4 \mathrm{~mm}$ mean duct diameter at age 40 , a $5 \mathrm{~mm}$ mean duct diameter at age 50 , a $6 \mathrm{~mm}$ mean duct diameter at age 60 , and so on" $[8,9]$. CBD size was adjusted according to age. In this study an "abnormal" MRCP was defined as a dilated biliary system.

\section{Statistical analysis}

We performed a binary logistic regression statistical analysis and chi-square test using IBM SPSS Statistics Version 23 (IBM Corp., New York).

\section{Results}

\section{Patient demographics}

Three hundred fifty-eight cases were included in this study. The remaining 69 cases were excluded because of incomplete data. This study had a female preponderance of $65 \%(n=231)$ compared with $35 \%$ male $(n=127)$. The mean age was 60 years (median $62, \mathrm{SD} \pm 18$ ) with a minimum and maximum age of 20 and 101 years, respectively, and an age range of 81 years.

\section{Clinical presentation}

The primary symptom in this cohort of patients was abdominal pain in $63 \%(n=225)$, with $20 \%$ having concurrent deranged serum LFTs $(n=44)$ and $8 \%$ jaundice $(n=18)$. In addition, $19 \%$ had isolated deranged LFTs $(n=42)$ and $12 \%$ painless jaundice $(n=27)$. Other clinical indications for imaging included pancreatitis $(n=31)$, weight loss $(n=8)$, abnormal previous imaging, most commonly CT $(n=9)$, and fever $(n=3)$ (Table 1).

\section{Imaging}

Initial index imaging was US of the abdomen in $79 \%$ of cases $(n=284)$ and CT of the abdomen in $21 \%(n=74)$. Initial index imaging with US or CT demonstrated a dilated biliary system $>6 \mathrm{~mm}$ in $68 \%$ of cases $(\mathrm{n}=245)$, with the remaining $32 \%$ $(n=113)$ having a normal caliber CBD. Of the latter 113 cases, 19 demonstrated a dilated CBD on MRCP (17\%). (Fig. 1,2). Since not all reports provided a numeric value for CBD diameter, a report stating "dilated CBD" or "extra-hepatic duct dilatation" was presumed to be $>6 \mathrm{~mm}$ after discussion with the reporting

Table 1 Clinical indications for initial imaging

\begin{tabular}{lcc}
\hline Clinical indication & Cases (n) & Overall \% \\
\hline Abdominal pain & 225 & 63 \\
Deranged liver function tests & 86 & 24 \\
\hline Clinical jaundice & 45 & 13 \\
Pancreatitis & 31 & 9 \\
Weight loss & 8 & 2 \\
\hline Abnormal previous imaging & 9 & 3 \\
\hline Fever & 3 & 1 \\
\hline
\end{tabular}




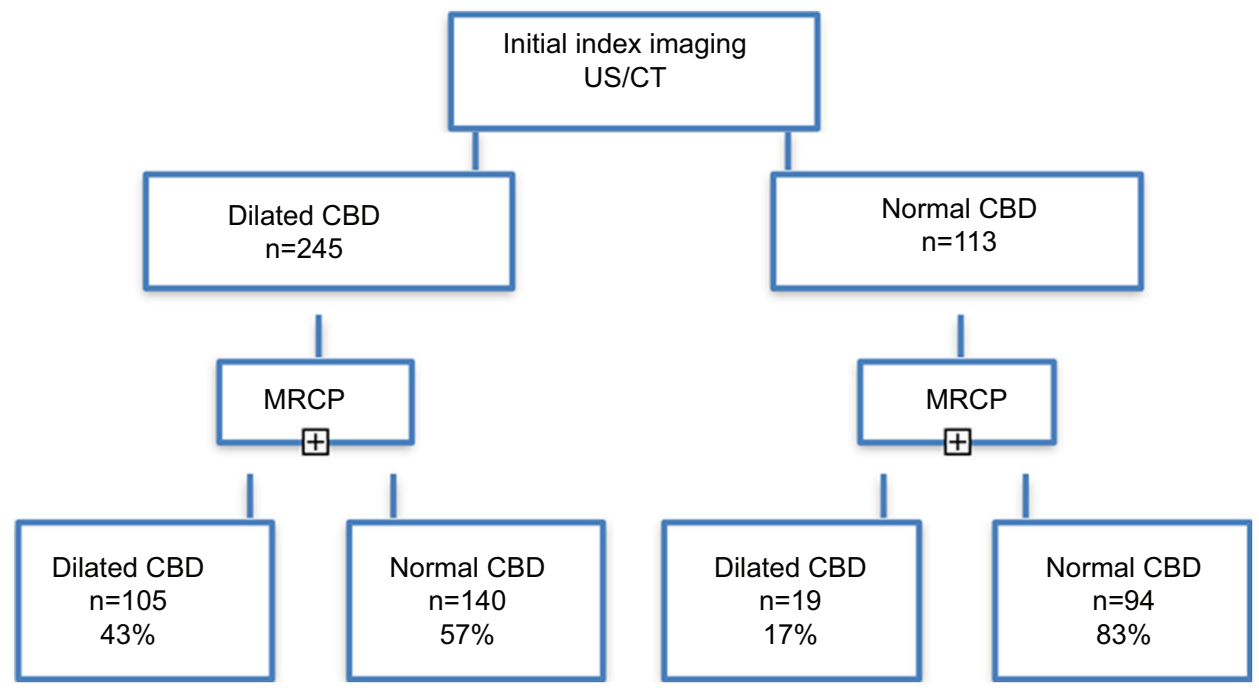

Figure 1 Flow chart demonstrating the diagnostic yield of an abnormal biliary tree on MRCP

US, ultrasound; CT, computed tomography; CBD, common bile duct; MRCP, magnetic resonance cholangiopancreatography

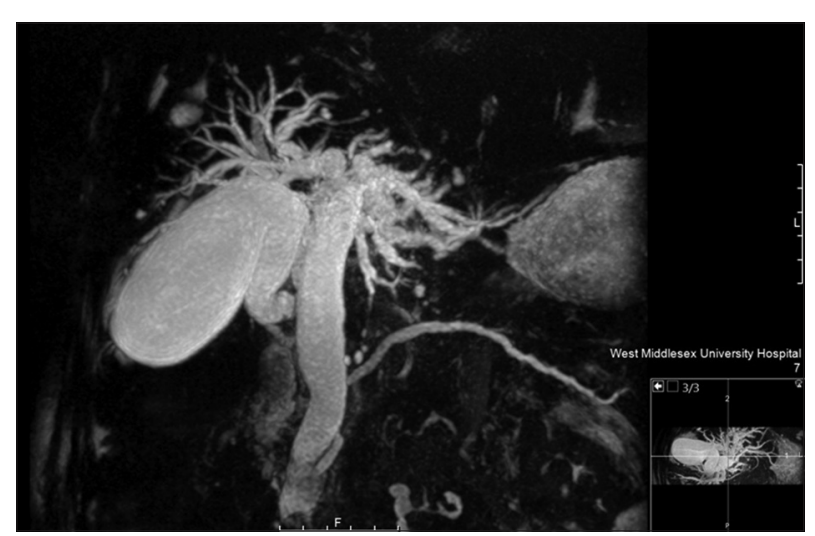

Figure 2 MRCP: a 77-year-old male presented with clinical jaundice and symptoms and biochemical analysis consistent with gallstone pancreatitis (bilirubin $69 \mu \mathrm{mol} / \mathrm{L}$, ALP $486 \mathrm{IU} / \mathrm{L}$ ). An initial ultrasound scan of his abdomen demonstrated a non-dilated CBD and no sonographic evidence of a calculus. Subsequent MRCP measured a $1.8 \mathrm{~cm}$ CBD containing multiple calculi. During the ERCP examination an $8 \mathrm{~mm}$ stone was removed with a balloon, sphincterotomy was performed and a CBD stent inserted. Subsequently, the patient had a laparoscopic cholecystectomy and removal of the CBD stent within 8 weeks of initial presentation

$M R C P$, magnetic resonance cholangiopancreatography; ALP, alkaline phosphatase; $C B D$, common bile duct; ERCP, endoscopic retrograde cholangiopancreatography

radiologists. Of the 69 cases excluded, 22 were due to difficulty visualizing the biliary tree on index imaging.

Abnormal index imaging was significantly associated with a dilated CBD on subsequent MRCP, accounting for $43 \%(n=104)$ of patients $(\mathrm{P}<0.001)$. Conversely, 113 patients underwent an MRCP with normal initial index imaging, of whom 19 were found to be positive for dilated ducts (17\%). In this cohort of 113 patients, 16 patients had an ALP level elevated to greater than 3 times the upper limit of normal, with 14 cases reaching 2-3 times the normal range and 39 up to double the normal range $(14 \%, 12 \%$, and $35 \%$, respectively). Of the first group, with the highest ALP levels, 50\% had an abnormal MRCP, followed by 2 cases (14\%) in the $2-3 \times$ elevation group and $4(10 \%)$ in those with up to double the normal range. We found ALP to be an independent positive predictor for an abnormal MRCP $(\mathrm{P}=0.003)$, whereas the remainder of the LFTs, such as bilirubin, AST, ALT and GGT, were not. Sex and age were also not significant (Table 2).

ALP was elevated to greater than 3 times the upper limit of normal in 34\% of MRCP cases with a dilated biliary system compared to $10 \%$ with a normal scan. An ALP twice the upper limit of normal was comparable between both groups: $13 \%$ of abnormal MRCP vs. $11 \%$ of normal MRCP outcomes.

We analyzed numerous independent variables to identify risk factors for a positively dilated CBD on MRCP. Abnormal index imaging, ALP and clinical jaundice were all significantly associated with a positive MRCP $(\mathrm{P}<0.001, \mathrm{P}=0.028$, and $\mathrm{P}=0.018$, respectively). Sex, age, remaining LFTs, and other clinical indications for the initial request were not related. A bilirubin of $>50 \mu \mathrm{mol} / \mathrm{L}$ is accepted as being the borderline for clinically detectable jaundice. There is a strong association between a raised bilirubin over $40 \mu \mathrm{mol} / \mathrm{L}$ in this case and a positively dilated $\mathrm{CBD}$ on MRCP $(\mathrm{P}=0.039)$ (Table 3$)$.

\section{Discussion}

Transabdominal US is recognized as the gold standard firstline investigation as it is noninvasive, relatively inexpensive, and readily available [1]. However the findings are operatordependent and although it has a specificity of 1.00 , the low sensitivity of 0.3 for identifying ductal stones means that as many as two of every three CBD stones are missed [1]. All reports should comment on dilatation of the biliary system, even if it is negative. If there is further suspicion of ductal 
Table 2 Factors associated with abnormal MRCP after normal index imaging (LFT values are considered abnormal)

\begin{tabular}{|c|c|c|c|c|c|}
\hline \multirow[t]{2}{*}{ Variable } & \multicolumn{2}{|c|}{ Cases (n) } & \multirow[t]{2}{*}{ P-value } & \multirow[t]{2}{*}{ Odds ratio } & \multirow[t]{2}{*}{$95 \%$ confidence intervals } \\
\hline & Normal MRCP & Abnormal MRCP & & & \\
\hline Sex & Female: 50 Male: 44 & Female: 13 Male: 6 & 0.573 & 1.417 & $0.421-4.763$ \\
\hline Age & $20-87$ & $38-78$ & 0.090 & 1.033 & $0.995-1.073$ \\
\hline Bili & 34 & 15 & 0.579 & 0.996 & $0.983-1.010$ \\
\hline ALP & 55 & 14 & 0.003 & 1.006 & $1.002-1.010$ \\
\hline AST & 49 & 13 & 0.284 & 1.002 & $0.998-1.007$ \\
\hline ALT & 62 & 13 & 0.407 & 0.998 & $0.994-1.002$ \\
\hline GGT & 65 & 14 & 0.611 & 1.000 & $0.998-1.001$ \\
\hline
\end{tabular}

MRCP, magnetic resonance cholangiopancreatography; LFT, liver function test; Bili, bilirubin, ALP, alkaline phosphatase; ALT, alanine transaminase; AST, aspartate aminotransferase; GGT, $\gamma$-glutamyltransferase

Table 3 Factors associated with positively dilated CBD on MRCP

\begin{tabular}{lcccc}
\hline Variable & Cases (n) & P-value & Odds ratio & 95\% confidence intervals \\
\hline Sex & Female: 79 Male: 44 & 0.7 & 0.902 & $0.533-1.526$ \\
Age & Range $20-101$ & 0.152 & 1.011 & $0.996-1.026$ \\
Abnormal index imaging & 104 & $<0.001$ & 0.292 & $0.160-0.533$ \\
Bili & 63 & 0.123 & 0.997 & $0.993-1.001$ \\
ALP & 84 & 0.028 & 1.002 & $1.000-1.004$ \\
AST & 70 & 0.486 & 0.999 & $0.997-1.002$ \\
ALT & 73 & 0.843 & 1.000 & $0.998-1.003$ \\
GGT & 95 & 0.495 & 1.000 & $0.999-1.001$ \\
Abdominal pain & 77 & 0.280 & 0.710 & $0.381-1.321$ \\
Clinical jaundice & 25 & 0.018 & 0.399 & $0.186-0.856$ \\
Pancreatitis & 6 & 0.964 & 0.974 & $0.313-3.029$ \\
Weight loss & 6 & 0.256 & 0.442 & $0.108-1.809$ \\
\hline Previous imaging & 1 & 0.306 & 3.268 & $0.338-31.585$ \\
Fever & 2 & 0.700 & 0.677 & $0.93-4.939$ \\
\hline
\end{tabular}

CBD, common bile duct; $M R C P$, magnetic resonance cholangiopancreatography; Bili, bilirubin, $A L P$, alkaline phosphatase; ALT, alanine transaminase;

AST, aspartate aminotransferase; GGT, $\gamma$-glutamyltransferase

stones in cases with abnormal LFTs or a US-confirmed dilated $\mathrm{CBD}$, according to UK guidelines an MRCP examination should be performed [1,5]. Endoscopic US is also an accepted but invasive imaging modality, albeit with limited resources. Elevated serum bilirubin $(>20 \mu \mathrm{mol} / \mathrm{L})$ and ALP $(>110 \mathrm{IU} / \mathrm{L})$ reflect an obstructed biliary system. US is superior to CT and is an excellent initial imaging modality for suspected bile duct pathology; it allows appropriate selection of patients who may warrant an MRCP. Our study demonstrates that normal index imaging reduces the risk of an abnormal CBD on MRCP by $70 \%$.

Over the past two decades, MRCP has become significantly morewidespreadasafundamentalimagingmodality, providingan alternative to endoscopic retrograde cholangiopancreatography (ERCP) for the investigation of both biliary and pancreatic disorders $[1,5,7,8]$. The heavily T2-weighted images allow for images with high signal intensity to diagnose filling defects, strictures or extraluminal compression $[7,9,10]$. For this reason, it precludes unnecessary instrumentation of the ducts with ERCP if the findings are negative. ERCP also carries a 5-30\% failure rate, with a morbidity of $1-7 \%$; hence, MRCP has proven to be cost-effective [10]. MRCP has a sensitivity and specificity of $85 \%$ and $93 \%$, respectively, in the detection of ductal stones as small as $2 \mathrm{~mm}$ [11]. It offers a noninvasive evaluation of the biliary system with accurate measurements of the CBD. Universally, a $4 \mathrm{~mm}$ CBD is considered a normal diameter, with an increase of $1 \mathrm{~mm}$ per decade after the age of 40 . The upper limit of normal is $6 \mathrm{~mm}$, but this is considered in relation to the patient's age $[12,13]$.

MRCP is not routinely indicated when a US demonstrates a CBD within normal limits. However, our study has shown that, apart from a normal US/CT CBD size, an elevated ALP level increases the risk of an abnormal CBD size on MRCP, with our study supporting a rise to 3 times the upper limit of normal. Therefore, if there is a high clinical suspicion of 
ductal pathology and an elevated serum ALP with normal US, an MRCP examination should be strongly considered as the next imaging modality of choice. This should be in the absence of other factors that may cause ALP elevation, such as bone disorders and pregnancy.

With the importance of financial justification in the NHS, expensive imaging modalities such as MRCP need to be based on appropriate clinical indications to increase the likelihood of gaining demonstrably useful information. The indications for imaging seen in this study were comparable to those in the literature. Our study reveals a reasonable diagnostic yield of $18 \%$ when considering performing an MRCP in patients with suboptimal visualization of the biliary tree on index imaging: $50 \%$ had raised associated bilirubin and ALP.

MRCP does allow more detailed investigation, creating the opportunity for alternative diagnoses, such as cholangiocarcinomas, ductal tumors and pancreatic disorders, as well as excluding these in patients who present with painless jaundice. Such diagnoses were seen in some of the patients in this study.

We selected several independent factors and analyzed the likelihood of returning an abnormal MRCP. We already know that abnormal index imaging, either US (excellent at measuring ductal size) or CT scan, is a good indication to progress to MRCP to help characterize the abnormality further. Traditionally, elevation of bilirubin and ALP has been associated with biliary obstruction; however, our study revealed a significant association only with elevated ALP. The remainder of the transaminases and GGT were not significant. From multiple LFTs on the system, we selected the results prior to initial US/CT imaging. In addition we looked at the relationship between the clinical indication for imaging and the outcome of the MRCP. Clinical jaundice was associated with an abnormal MRCP. These findings tell us that an abnormal CBD on US/ CT, elevated ALP and clinical jaundice are most likely to return an abnormal MRCP with a dilated biliary system. However, in clinical practice, it is recognized and established that US in the first instance is the best form of imaging of the biliary system. It is more readily accessible, less time-consuming, inexpensive, and gives immediate results. Interestingly, elevated bilirubin was not associated with an abnormal MRCP, whereas clinical jaundice was. Traditionally, clinical jaundice is evident with a bilirubin level above $50 \mu \mathrm{mol} / \mathrm{L}$. We would expect both to have the same relationship with abnormal MRCP findings. This discrepancy may have been due to clinical jaundice being described subjectively by different clinicians. Interestingly our analysis demonstrates a likelihood of a positively dilated CBD with a bilirubin $>40 \mu \mathrm{mol} / \mathrm{L}$.

Limitations to this study include the numerous indications for imaging requests. These were not standardized and the indication was variable according to the clinical information volunteered by the clinician writing the request form, many of whom were junior doctors. For example, we were unable to differentiate between abdominal pain and pancreatitis. In addition fever was listed in several cases: it is possible, however, that this may have been a symptom for other patients but was not volunteered on the request form. In addition, this study was performed retrospectively for a group of patients investigated with MRCP and we do not have a control group to compare our cohort with.

Statistically we have demonstrated that it is efficacious to proceed to MRCP with abnormal findings on index imaging, clinical jaundice or elevated serum ALP. However other clinical indices, such as pain or abnormal liver chemistry (other than raised ALP), are not independently predictive of a positive finding on MRCP. In the context of normal CBD size on index imaging, having a concurrent elevated ALP is a predictor of positive MRCP findings.

This study has identified that there is a reasonable diagnostic yield for MRCP in the absence of a dilated biliary system on previous US/CT. We propose that there should be a specific rationale for performing MRCP when initial imaging is normal. This study recommends that MRCP should be strongly considered in the context of an elevated ALP and normal US/CT biliary system. In addition, we suggest a bilirubin threshold of $>40$ $\mu \mathrm{mol} / \mathrm{L}$ can be used as an indicator of clinical jaundice.

\section{Summary Box}

\section{What is already known:}

- Cholelithiasis is increasingly common, with a UK prevalence of $15 \%$

- $10-20 \%$ of the $15-26 \%$ of patients with symptomatic gallstones will progress to develop choledocholithiasis

- UK NICE guidelines recommend the use of magnetic resonance cholangiopancreatography (MRCP) in cases of abnormal liver function tests or a dilated common bile duct (CBD)

\section{What the new findings are:}

- Normal index imaging reduces the risk of an abnormal CBD on MRCP by $70 \%$

- A dilated CBD on index imaging, clinical jaundice or elevated alkaline phosphatase (ALP) are independently associated with abnormal MRCP findings

- MRCP should be strongly considered if there is a normal biliary system on ultrasound or computed tomography in combination with an elevated ALP

- Elevated bilirubin $>40 \mu \mathrm{mol} / \mathrm{L}$ is significantly associated with a dilated $\mathrm{CBD}$ on $\mathrm{MRCP}$

\section{References}

1. NICE clinical guideline 188, Gallstone Disease. October 2014. https://www.nice.org.uk/guidance/cg188.

2. Chandrasekhara V, Ginsburg GG. Gallstones and biliary tract disease. In: A Rustgi (Editor): ACP Medicine Gastroenterology. Scientific Am Med. Decker: Ontario, Canada 2013; pp 1-15.

3. iRefer, Royal College Radiology Guidelines. https://www.irefer.org.uk Accessed 2016 
4. Abraham S, Rivero HG, Erlikh IV, Griffith LF, Kondamudi VK. Surgical and nonsurgical management of gallstones. Am Fam Physician 2014;89:795-802.

5. Williams EJ, Green J, Beckingham I, Parks R, Martin D, Lombard M; British Society of Gastroenterology. Guidelines on the management of common bile duct stones (CBDS). Gut 2008;57:1004-1021.

6. Tse F, Barkun JS, Romagnuolo J, et al. Nonoperative imaging techniques in suspected biliary tract obstruction. HPB (Oxford) 2006;8:409-425.

7. Griffin N, Charles-Edwards G, Grant LA. Magnetic resonance cholangiopancreatography: the ABC of MRCP. Insights Imaging 2012;3:11-21.

8. Laing FC. The gallbladder and bile ducts. In: Rumack C, Wilson S, Carboneau JW (editors): Diagnostic ultrasound. Mosby: St Louis, Mo; 1998, pp.175-223.
9. Pickuth D. Radiologic diagnosis of common bile duct stones. Abdom Imaging 2000;25:618-621

10. Mibang N, Hajong R, Tongper D, et al. The role of magnetic resonance cholangiopancreaticography (MRCP) in predicting common bile duct stone in symptomatic gallstone disease. IOSRJDMS 2013;6:84-90. URL: http://www.iosrjournals.org/iosr-jdms/ papers/Vol6-issue6/N0668490.pdf

11. Horrow MM. Ultrasound of the extrahepatic bile duct: issues of size. Ultrasound Q 2010;26:67-74.

12. Perret RS, Sloop GD, Borne JA. Common bile duct measurements in an elderly population. J Ultrasound Med 2000;19:727-730.

13. Bowie JD. What is the upper limit of normal for the common bile duct on ultrasound: how much do you want it to be? Am J Gastroenterol 2000;95:897-900. 